\title{
Title: Developmental dynamics of cross-modality in mouse visual cortex
}

\author{
Authors: Ryoma Hattori ${ }^{1,2}$ and Takao K Hensch ${ }^{1 *}$
}

\author{
Affiliations: \\ ${ }^{1}$ Center for Brain Science, Department of Molecular \& Cellular Biology, Harvard University, 52 \\ Oxford St, Cambridge, MA 02138 USA \\ ${ }^{2}$ Neurobiology Section, Center for Neural Circuits and Behavior, Department of Neurosciences, \\ University of California, San Diego, La Jolla, CA 92093, USA \\ *Correspondence to: hensch@mcb.harvard.edu
}

\section{SUMMARY}

Maturation of GABAergic circuits in primary visual cortex (V1) opens a critical period (CP), a developmental window of enhanced plasticity for visual functions. However, how inhibition promotes the plasticity remains unclear. Here, we investigated the developmental dynamics of auditory responses and audiovisual interactions in mouse V1. Modulation of V1 spiking activity by a transient sound was temporally dynamic with alternating enhancement and suppression phases. When paired with grating visual stimuli, sound-driven spike enhancement and suppression were weaker and stronger with preferred orientation than with non-preferred orientations, respectively, leading to impaired net orientation selectivity in V1 neurons. Strikingly, the net orientation selectivity was impervious to sound specifically during the CP due to equal total amounts of sound-driven spike enhancements and suppressions. This balance of spike modulations at the $\mathrm{CP}$ was achieved by the preferential maturation of sound-driven spike suppression. However, further maturation of sound-driven spike enhancement broke the balance after the CP. Spectral analysis of field potentials revealed the enhancement of a GABA-mediated sound-driven power suppression specifically at CP. Reduction of inhibition by 10-day darkexposure or genetic deletion of GAD65 gene dampened sound-driven spike suppression in V1. Furthermore, acute suppression of either parvalbumin-expressing (PV) or somatostatinexpressing (SST) neurons suggested their early or late recruitments by sound, respectively. Our results point to the dampened net non-visual sensory influence as one of the functional roles of GABA circuit maturation during a developmental CP. The insensitivity of visual selectivity to sound during the $\mathrm{CP}$ may promote functional maturation of $\mathrm{V} 1$ as visual cortex.

\section{INTRODUCTION}

Neural circuits of sensory cortex are shaped by sensory experience in postnatal life. This experience-dependent plasticity is heightened during an early developmental period, called critical period (CP). The properties and underlying mechanism of $\mathrm{CP}$ has been studied most extensively in primary visual cortex (V1). For example, brief visual deprivation of one eye during the $\mathrm{CP}$ dampens the cortical responsiveness to deprived eye stimulation (Espinosa \& Stryker, 2012). In mice, the CP of V1 is between P21 and P35 with its peak sensitivity to monocular deprivation around P28 (Gordon \& Stryker, 1996), and the plasticity gradually 
declines after the period and is almost absent around P110 (Lehmann \& Löwel, 2008).

Cortical inhibition matures during CP (Lazarus \& Huang, 2011), and accumulating evidence indicates that the maturation of cortical inhibitions triggers the opening of CP (Espinosa \& Stryker 2012; Hensch, 2005). Mice lacking a synaptic isoform of GABA synthase (GAD65 KO) do not open their CP unless their cortical inhibition is rescued by benzodiazepine treatment (Hensch et al., 1998; Fagiolini \& Hensch, 2000; Iwai et al., 2003; Kanold et al., 2009). Similarly, cortical infusion of benzodiazepine opens a CP even before the normal onset of CP in wild-type mice (Fagiolini \& Hensch, 2000). Other manipulations that indirectly affect the maturational state of cortical inhibition also shifts the timing of CP (Huang et al, 1999; Hanover et al., 1999; Gianfranceschi et al., 2003; Di Cristo et al., 2007; Sugiyama et al., 2008; Kobayashi \& Hensch, 2015). The mechanism as to how mature cortical inhibitions enhance plasticity for visual functions has been speculative, but a recent study suggested that preferential suppression of spontaneous activities relative to visually evoked activities might enhance visual plasticity by increasing signal-to-noise $(\mathrm{S} / \mathrm{N})$ ratio of visual inputs (Toyoizumi et al., 2013). However, the source of such noise that is dampened during CP has been unknown.

Although V1 predominantly receives and processes visual signals coming from thalamus, it also exhibits non-visual sensory activities such as auditory and somatosensory-related signals (Wallace et al., 2004; Iurilli et al., 2012; Vasconcelos et al., 2011; Liang et al., 2013; Romei et al., 2009; Romei et al., 2012; Rohe \& Noppeney, 2016). Anatomical tracing studies revealed direct neural projections from auditory cortex to V1 (Charbonneau et al., 2012; Lu et al., 2014; Kim et al., 2015), and transection between auditory cortex and V1 abolishes sound-evoked responses in V1 in vivo (Iurilli et al., 2012). These studies suggest that multisensory interaction already occurs between primary sensory cortical areas. However, how different sensory inputs interact in primary sensory cortex remains largely unexplored. Furthermore, it is unknown how non-visual sensory activities are regulated during early postnatal life when neural circuitry undergoes extensive experience-dependent plasticity.

In this study, we investigated the impact of sound on visual responses in mouse V1 at different postnatal ages by electrophysiology in vivo. We found that sound-driven modulation of visual spiking activities consists of temporally dynamic oscillatory phases and depended on the orientation of concurrent grating stimuli. This sound-driven modulation of visual response impaired orientation selectivity of V1 neurons except during CP when total amounts of sounddriven spike enhancement and suppression were equalized. We further showed how local inhibitory circuits regulate the sound impacts on V1. The developmental dynamics of crossmodality and its regulation by inhibitory circuitry hint at the mechanism underlying the inhibitory control of CP plasticity.

\section{RESULTS}

\section{Temporally dynamic sound-driven spike modulations and their dependence on concurrent visual orientation}

Neurons in V1 preferentially respond to specific orientation of visual stimuli (Niell \& Stryker, 2008). This orientation selectivity of V1 neurons allowed us to assess the sound-driven spike modulations as a function of the magnitude of concurrent visual spiking activities in each neuron. To vary the efficacy of visual inputs to individual cells during spike recordings in vivo, drifting gratings of different orientation were used as visual stimuli with or without concurrent 
white noise (WN) sound (Figure 1A). In our studies, we presented WN sound only for the first $500 \mathrm{~ms}$ of $3 \mathrm{sec}$ visual stimulus period to examine the influence of a transient sound on visual image.

We found that the earliest sound-driven spike modulation emerges as an enhancement even before the onset of visual spiking activities (P70-90, 149 cells, peak latency of $27.2 \pm 0.9 \mathrm{~ms}$, mean \pm SEM) (Figures 1B, 1C, 2 and S1). The fast latency of auditory response in V1 is consistent with its short latency in auditory cortex (Linden et al., 2003). This transient sounddriven spike enhancement at pre-visual phase was immediately followed by a tri-phasic sounddriven modulation of visually-evoked activities, which depended on the grating orientation and persisted even beyond the sound stimulus (Figures 1B, 1C and 2). During the early (50-130 ms) and late (1-3 s) phases after stimulus onset, sound preferentially suppressed visual responses evoked by the preferred orientation. In contrast, sound preferentially enhanced visual responses evoked by the non-preferred orientation during a middle phase. The preferential multisensory enhancement with less effective orientation is consistent with the 'inverse effectiveness rule' of multisensory integration in classically defined multisensory areas such as superior colliculus (Stein \& Wallace, 1996). However, unlike in multisensory areas, the inverse effectiveness extends to suppressive range at early (50-130 ms) and late (1-3 s) phases in V1, a classically defined unisensory area. We confirmed that the multisensory suppression is expected in areas with strong unimodality using divisive normalization model (Figure S2) which has successfully simulated multisensory integration in classically defined multisensory areas (Carandini \& Heeger, 2011; Ohshiro et al., 2011).

\section{Sound-driven spike modulations are balanced specifically during $\mathbf{C P}$}

The impact of non-visual sensory inputs on V1 can be most severe when the neural circuits undergo experience-dependent plasticity for their functional maturation. Thus, we compared the sound modulations of V1 spiking activities among pre-CP (P17-19, 141 cells), CP (P26-31, 108 cells) and post-CP (P70-90, 149 cells) groups. In all age groups, a transient spike enhancement at pre-visual phase was followed by a tri-phasic modulation of visual spiking activities (Figure 2) but with different suppression/enhancement ratios (Figure 3A). The early (50-130 ms) and late (1-3 s) spike suppressions matured and reached a plateau level during CP. In addition to the magnitude maturation, the latency of early spike suppression became faster during CP (Figure $3 \mathrm{~B})$. On the other hand, the middle phase spike enhancement (130-1000 ms) continued to mature even after CP (Figure 3A).

We then examined the net impact of sound on visual response by averaging the interaction throughout the period of visual presentation $(0-3 \mathrm{sec})$. We quantified the percent sound-driven modulation of visual response by multisensory index $(\mathrm{MSI}=[(\mathrm{VA}-\mathrm{V}) / \mathrm{V}] \times 100)($ Holmes \& Spence, 2005), and found that the MSI was transiently dampened during CP (Figure 3C). Sounddriven modulations of V1 spontaneous activities without concurrent visual stimuli similarly dampened their net impact during CP (Figure S1). These results indicate that sound-driven spike suppressions preferentially mature relative to enhancement during $\mathrm{CP}$ to offset the net modulation of V1 spiking activities. 
A

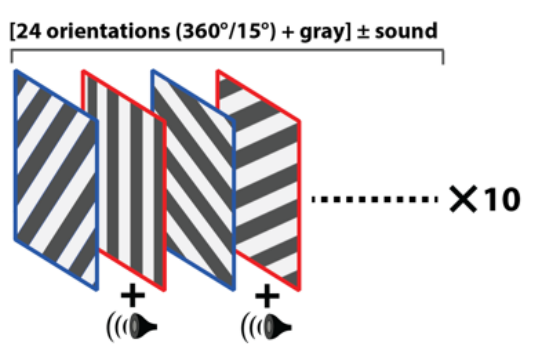

B

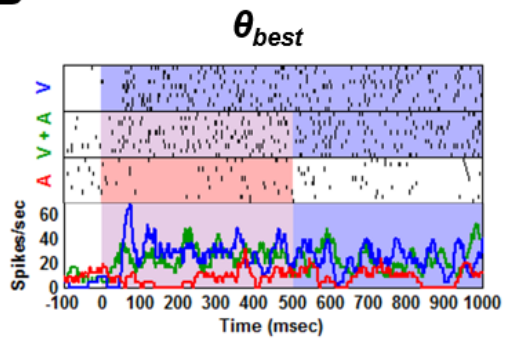

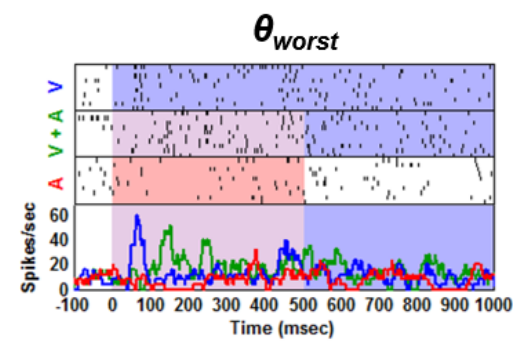

C

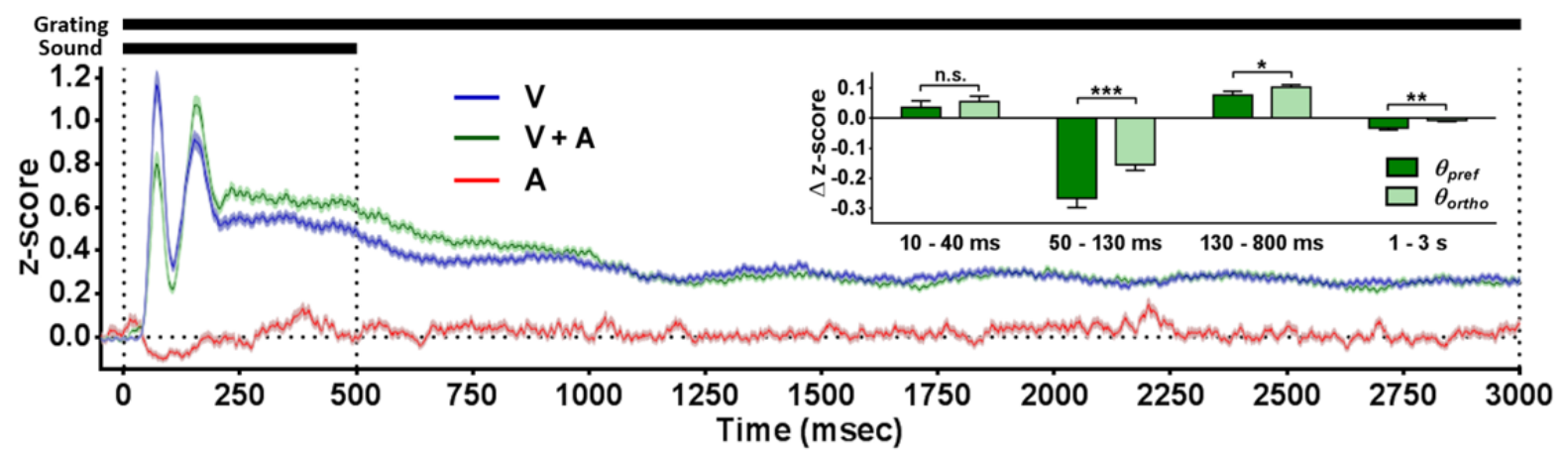

Figure 1. Sound-driven spike modulations and their dependence on visual stimuli in adult V1.

(A) Schematic of sensory stimulation paradigm. Drifting gratings with 24 different orientations $\left(360^{\circ} / 15^{\circ}\right)$ were presented in randomized order alternately with or without a WN sound. $\sim 10$ trials were averaged for each condition.

(B) Raster plots and peri-stimulus time histograms (PSTHs) of a typical representative cell in response to visual $(\mathrm{V})$, auditory $(\mathrm{A})$, and combined $(\mathrm{V}+\mathrm{A})$ stimuli. Audiovisual interactions with either the orientation that evoked the largest visual response $\left(\theta_{\text {best }}\right)$ or with the orientation that evoked the smallest visual response $\left(\theta_{\text {worst }}\right)$ in this example cell are shown.

(C) z-scored population averages (mean \pm SEM) of PSTHs (P70-90; 149 cells). PSTHs at all presented orientation were averaged for each cell. Inset shows $\Delta \mathrm{z}$-scores between visuo-auditory and visual conditions at each post-stimulus interval with preferred $\left(\theta_{\text {pref }}\right)$ or orthogonal orientation $\left(\theta_{\text {ortho }}=\theta_{\text {pref }} \pm 90^{\circ}\right)$ grating orientation. All error bars are SEM. Paired t-test, ${ }^{*} \mathrm{P}<$ $0.05, * * \mathrm{P}<0.01, * * * \mathrm{P}<0.001$. 


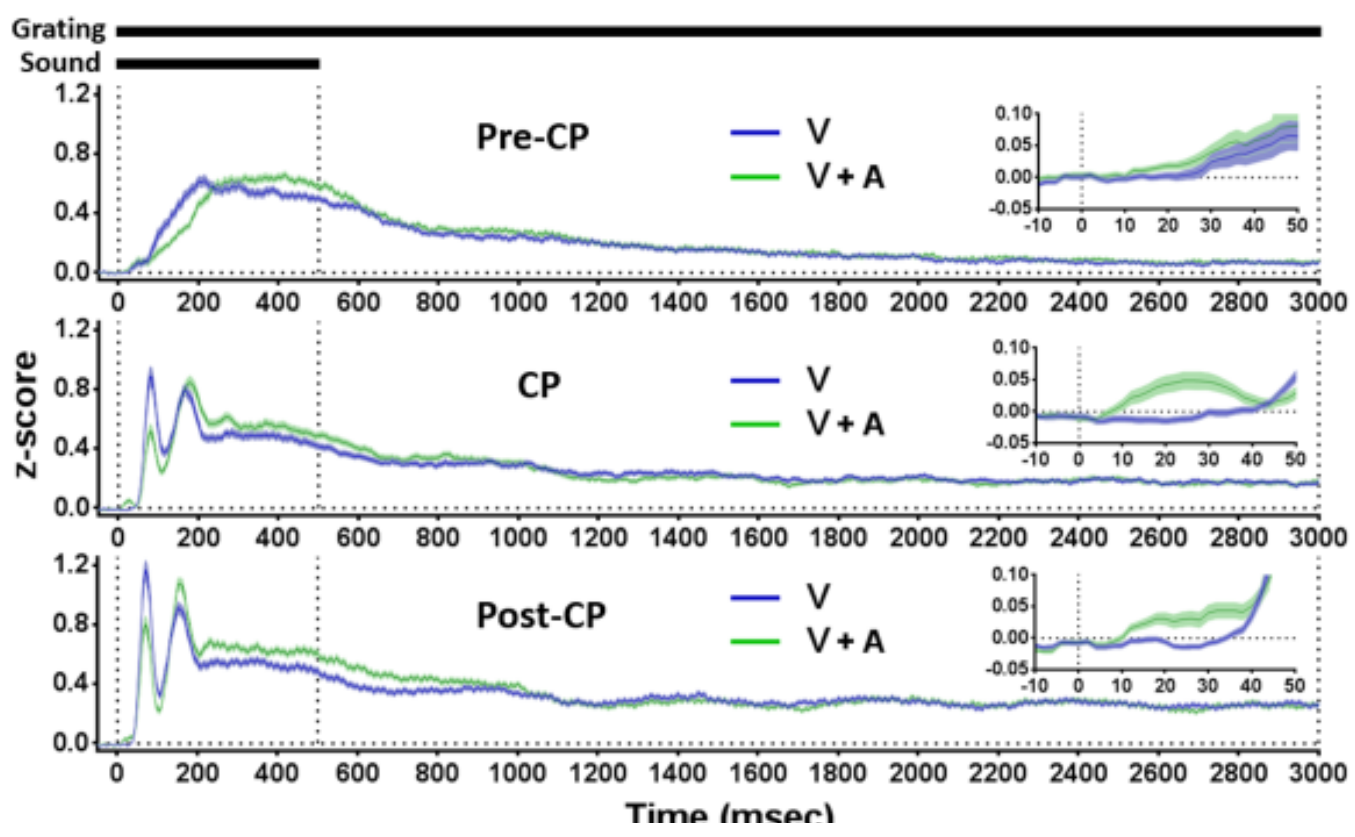

Figure 2. Sound-driven modulations of visual spiking activities at different postnatal periods.

Z-scored population averages (mean \pm SEM) of PSTHs from pre-CP (P17-19, 141 cells), CP (P26-31, 108 cells), and post-CP (P70-90, 149 cells) groups. PSTHs at all presented orientation were averaged for each cell. Inset highlights the pre-visual phase. 

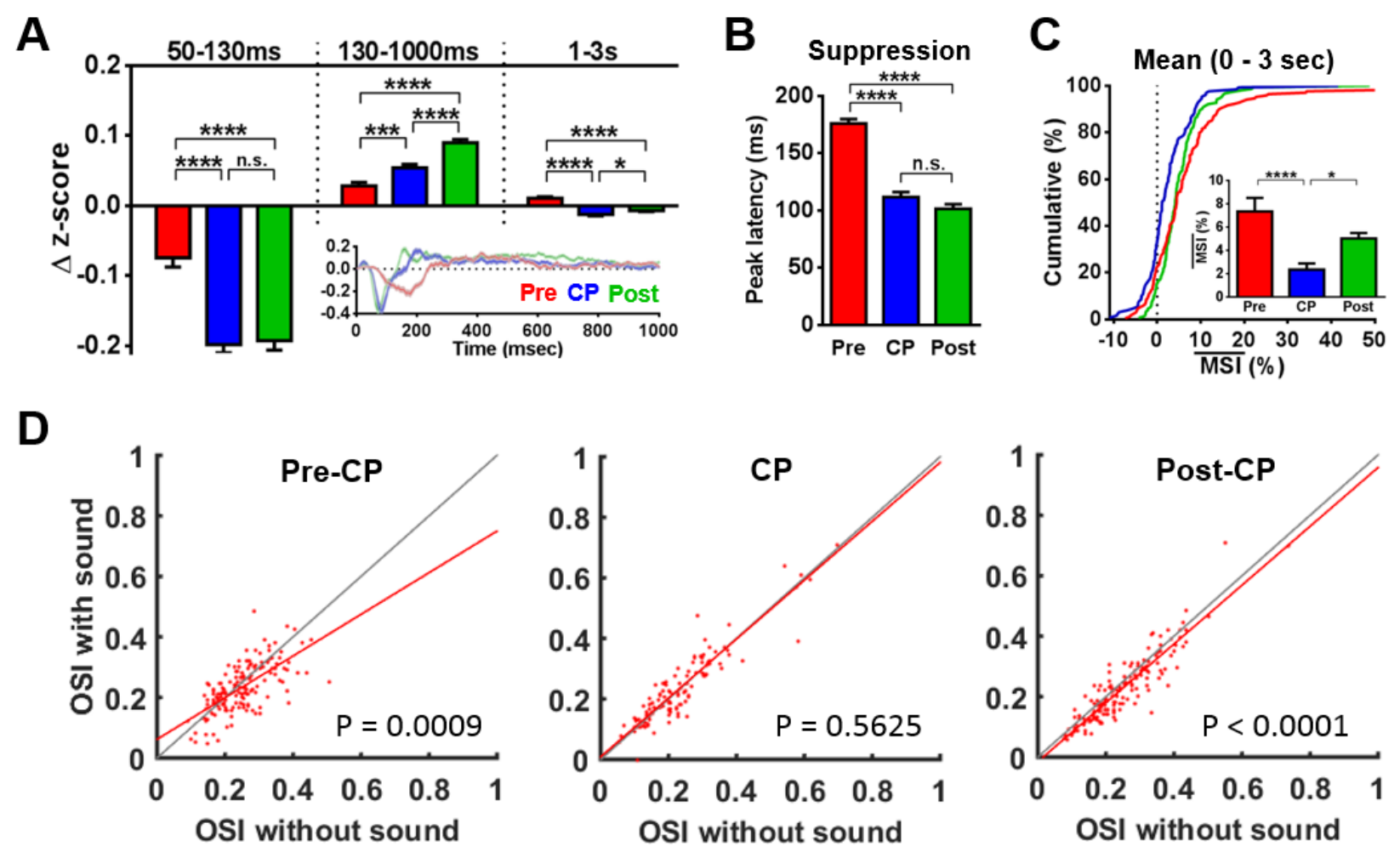

Figure 3. Balanced sound-driven spike modulations preserve orientation selectivity during CP.

(A) $\Delta z$-scores between visuo-auditory and visual conditions at different post-stimulus time intervals for different age groups. Inset shows the $\Delta \mathrm{z}$-scores as a function of time (mean \pm SEM). One-way ANOVA, followed by Holm-Sidak test.

(B) The peak latency of early sound-driven spike suppression. One-way ANOVA, followed by Holm-Sidak test.

(C) Mean of multisensory index (MSI) at all presented orientations. Kruskal-Wallis test, followed by Dunn's test.

(D) Scatter plots indicating OSI with and without concurrent sound stimuli. Least-squares regression lines of data points are colored in red. Paired t-test.

All error bars are SEM. $* \mathrm{P}<0.05, * * * \mathrm{P}<0.001, * * * * \mathrm{P}<0.0001$. 
Next, we examined the impact of sound on the orientation selectivity of V1 neurons. We found that sound impairs orientation selectivity index (OSI) of visual response in pre-CP and post-CP mice (Figure 3D). The impaired OSI is expected given the larger suppressions and smaller enhancement at preferred orientation than at non-preferred orientation (Figures 1C, S2A and S2B). On the other hand, sound did not impair OSI in CP mice (Figure 3D) due to the balanced sound-driven suppression and enhancement (Figures 3A, 3C, S1B and S1C).

\section{Developmental dynamics of sound-driven cortical oscillations}

Having characterized the developmental dynamics of sound-driven spike modulations, we next examined the gross impact of sound on V1 neural network by recording local field potentials. We used a brief $100 \mathrm{~ms}$ WN sound to induce a cross-modal auditory-evoked field potential (cAEP) in L2/3 of V1. Comparison among various age groups revealed unique shifts in the average waveform morphology of the cAEP at the beginning $(\sim \mathrm{P} 21)$ and after the end $(\sim \mathrm{P} 35)$ of CP (7 mice for P17-18; 5 mice for P20; 7 mice for P22; 6 mice for P24; 5 mice for P27; 12 mice for P31-32; 16 mice for P71-86; 5 mice for P208-295). Before and after this CP, a characteristically large negative peak (N1) with little or no delayed positive peak (P1) was observed, while a large P1 without an early N1 emerged during the CP (Figure 4A). Spectral analysis of cAEP revealed that N1 and P1 enhances and suppresses the power of cortical oscillations at wide frequency range, respectively, and the magnitudes of power modulations correlated with the amplitudes of their peaks (Figure 4B).

Given the maturation of cortical inhibitions in V1 during CP (Lazarus \& Huang, 2011), we asked whether the sound-driven power suppression of cortical oscillations depends on GABAmediated inhibitions. We found that the amplitude of P1 was diminished in GAD65 KO mice at CP (P27-31) (Figure 5A). However, their P1 amplitude could be fully recovered by acute local bath application of GABA-A receptor agonist, diazepam, onto V1 (Figure 5B). Further comparison of cAEPs at post-CP (P70-90) revealed the loss of P1 and enhancement of N1 in GAD65 KO mice (Figure 5C). We also recorded from mice that were exposed to complete darkness for 16-19 days from P60 (DE mice) and found the similarly diminished P1 and enhanced N1 (Figure 5C). Two weeks of dark-exposure dampens GABA-mediated inhibitions in adult rodent V1 (He et al., 2010; Huang et al., 2010; Stodieck et al., 2014). Thus, these results suggest that inhibitory circuits regulate the relative ratio of sound-driven power suppression/enhancement in V1, and their maturation at CP is responsible for the increased sound-driven power suppression at $\mathrm{CP}$. 

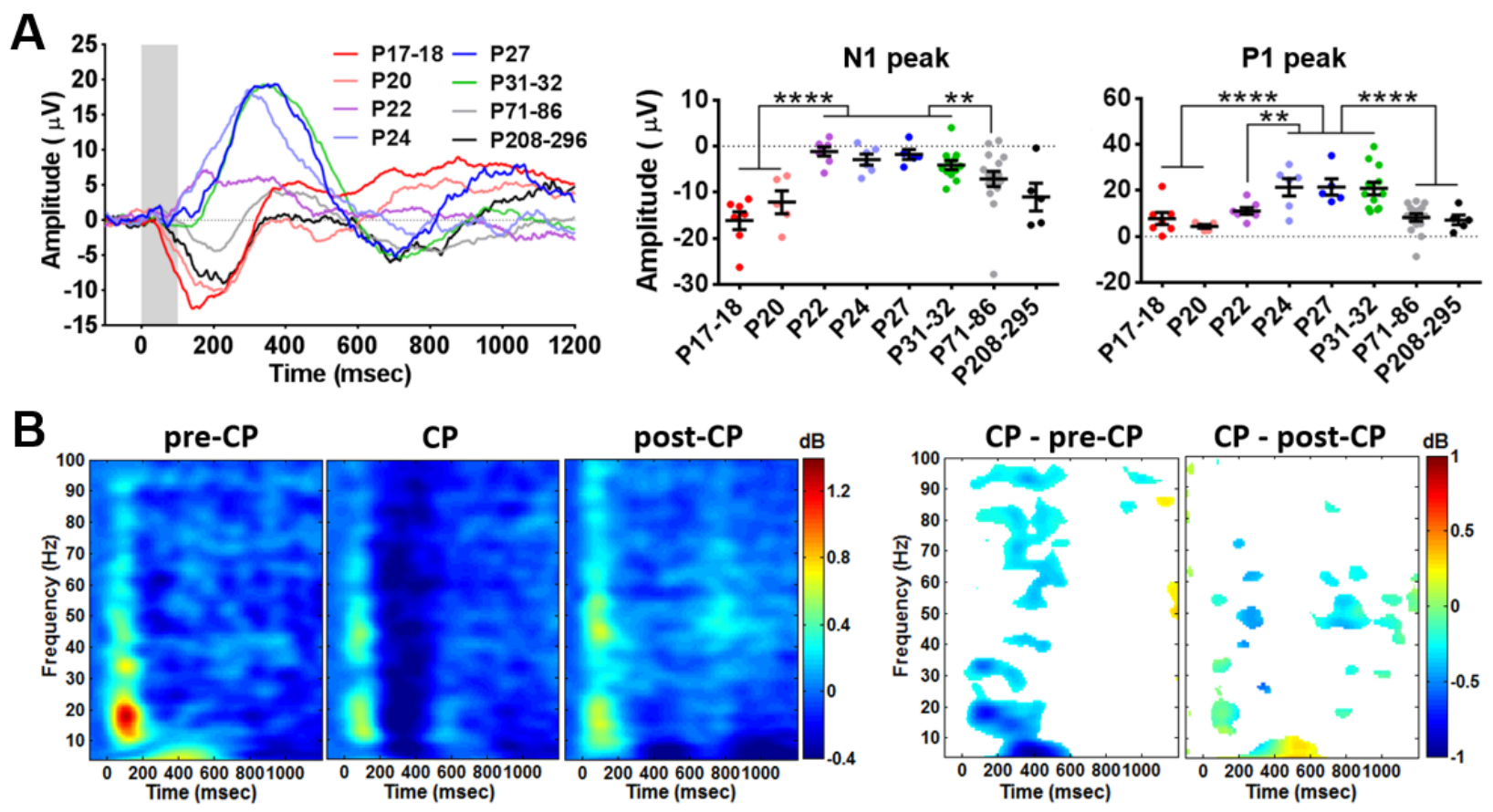

Figure 4. Sound-driven power suppression of cortical oscillations is specifically enhanced during CP.

(A) Average cAEP traces and N1/P1 amplitudes from L2/3 across postnatal days ( 7 mice for P17-18; 5 mice for P20; 7 mice for P22; 6 mice for P24; 5 mice for P27; 12 mice for P31-32; 16 mice for P71-86; 5 mice for P208-295). Shading indicates WN stimulus duration. One-way ANOVA, followed by Holm-Sidak test. All error bars are SEM. $* * \mathrm{P}<0.01$, ****P $<0.0001$. (B) Average spectral perturbation from baseline (pre-stimulus $500 \mathrm{~ms}$ period) after $\mathrm{WN}$ presentation at pre-CP (P17-20; 12 mice), $\mathrm{CP}$ (P24-32; 23 mice), and post-CP (> P70; 21 mice), and the statistics for the comparisons among pre-CP, $\mathrm{CP}$, post-CP groups. The areas with significant difference $(\mathrm{P}<0.05$, pixel-by-pixel ANOVA, followed by Holm-Sidak test) are colored, and the color values indicate their difference in $\mathrm{dB}$. 

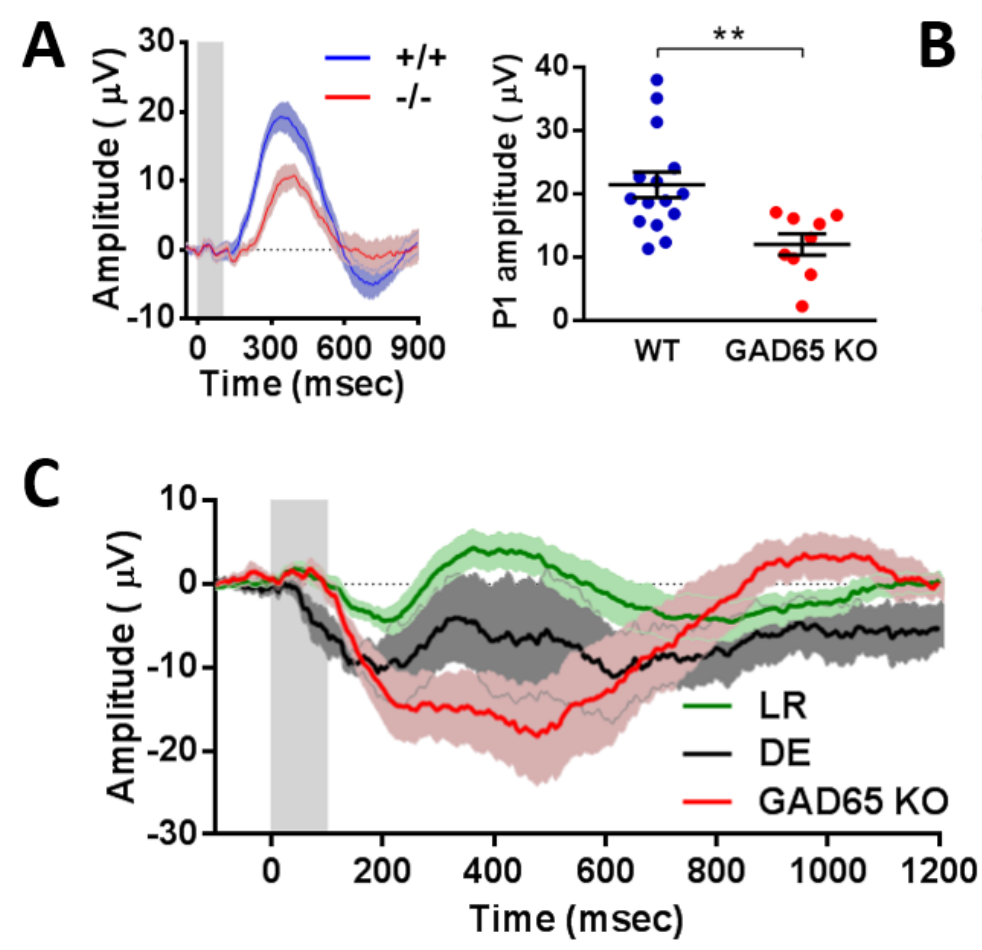
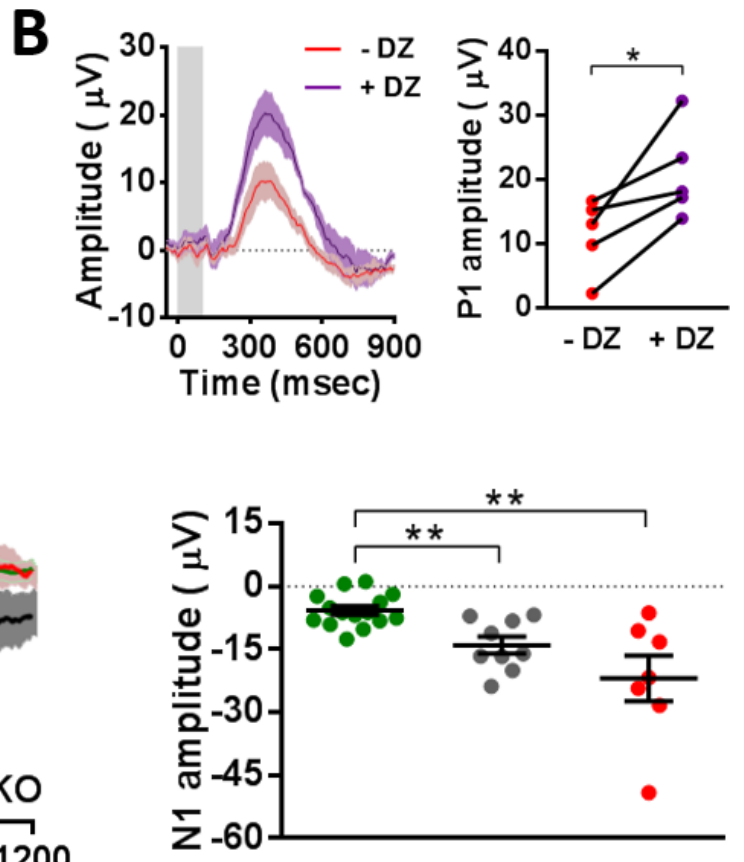

Figure 5. Waveform of cross-modal auditory evoked field potential depends on GABAmediated inhibition and visual experience.

(A) Average cAEP traces and P1 amplitudes from the L2/3 of WT (15 mice) and GAD65 KO (9 mice) at normal CP age (P27-31). Unpaired t-test.

(B) cAEP traces and P1 amplitudes before and after acute bath application of diazepam (DZ) on the V1 of GAD65 KO mice (5 mice, P30-31). Paired t-test.

(C) cAEP traces from LR (light-reared control: 15 mice), DE (dark-exposed: 9 mice) and GAD65KO (7 mice) at normal post-CP age (P70-90), and their N1 amplitudes. One-way ANOVA, followed by Holm-Sidak test.

All error bars are SEM. $* \mathrm{P}<0.05, * * \mathrm{P}<0.01$. 


\section{Inhibitory control of sound-driven spike modulations in V1.}

We next examined the role of cortical inhibitions in sound-driven spike modulations in V1. First, we compared sound-driven modulations of visual spiking activities among wild-type mice with or without dark-exposure (17-18 days of dark from P60, 201 cells) and normally reared GAD65 KO mice (156 cells) at adult age (P70-90). Although the middle phase sound-driven spike enhancement (130-1000 ms) was similar across three groups, early sound-driven spike suppression (50-130 ms) was slower and weaker in both DE and GAD65 KO mice (Figures 6A and $6 \mathrm{~B})$. In addition, late sound-driven spike suppression (1-3 s) was significantly impaired in GAD65 KO mice (Figure 6A). These diminished sound-driven spike suppressions in DE and GAD65 KO mice increased their overall MSIs (Figure 6C).

Soma-targeting parvalbumin (PV)-expressing and dendrite-targeting somatostatin (SST)expressing interneurons are the two major inhibitory cell types and functionally mature during the CP in V1 (Lazarus \& Huang, 2011). To investigate the contributions of each cell type on sound-driven modulations of visual spiking activities, we used mice expressing Archaerhodopsin (Arch), a light-gated $\mathrm{H}^{+}$-pump (Chow et al., 2010), in either PV- (PV-Cre x Ai35) or SST-cells (SST-Cre $\mathrm{x}$ Ai35) to optically suppress their activities in V1 during sound presentation (Figure 6D). PV-cell suppression decreased the early sound-driven spike suppression (50-130 ms), whereas SST-cell suppression enhanced it likely by disinhibiting PV-cells (Pfeffer et al., 2013). Furthermore, SST-cell suppression increased the sound-driven spike enhancement at middle phase (130-1000 ms) and decreased the late sound-driven spike suppression (1-3 s). These results suggest that the early sound-driven spike suppression is primarily mediated by PV-cells, but SST-cells mediate the late sound-driven spike suppression and limit the amount of sound-driven spike enhancement at the middle phase during audio-visual interactions in V1. 
A

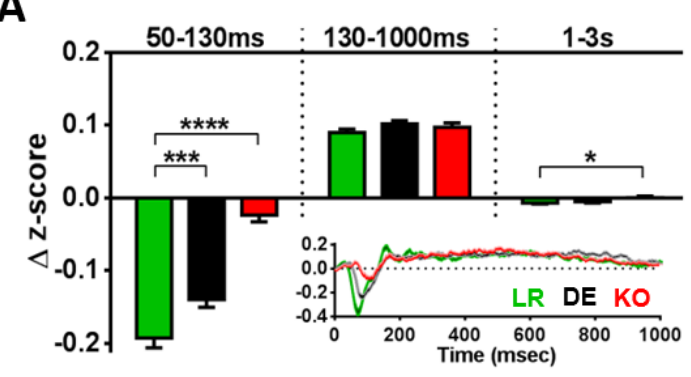

B

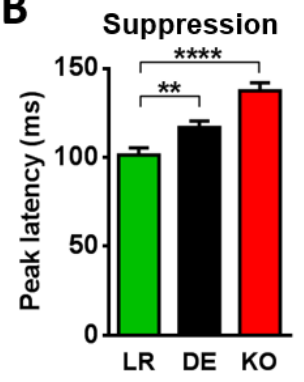

C

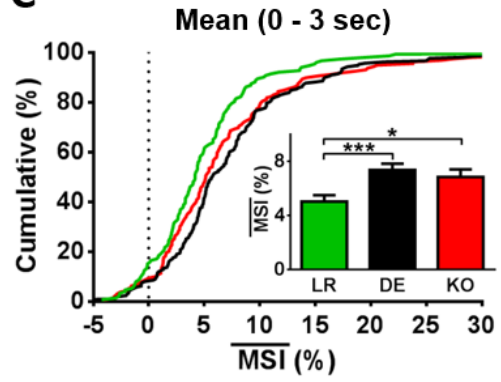

D

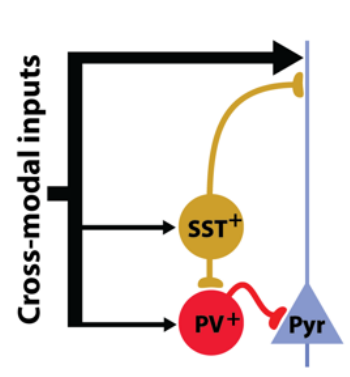

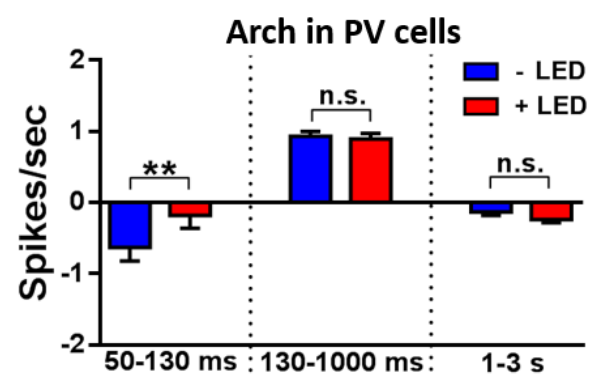

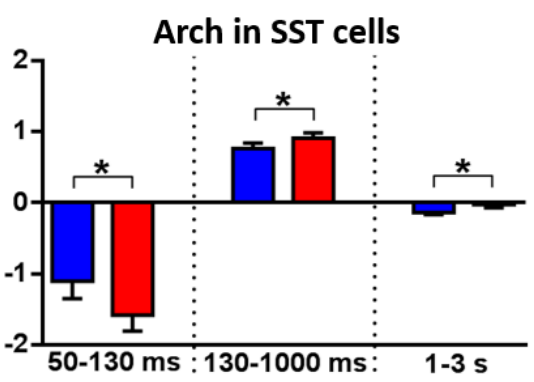

Figure 6. Inhibitory control of sound-driven spike modulations in V1.

(A) $\Delta z$-scores between visuo-auditory and visual conditions at different post-stimulus time intervals for LR (149 cells), DE (201 cells), GAD65 KO (156 cells) groups. All animals were recorded at normal post-CP age (P70-90). Inset shows the $\Delta \mathrm{z}$-scores as a function of time (mean \pm SEM). One-way ANOVA, followed by Holm-Sidak test.

(B) The peak latency of early sound-driven spike suppression. One-way ANOVA, followed by Holm-Sidak test.

(C) Mean of multisensory index (MSI) at all presented orientations. Kruskal-Wallis test, followed by Dunn's test.

(D) Sound modulations of visual spiking activities with or without suppression of PV-expressing (140 cells) or SST-expressing (145 cells) cells. All animals (PV-Cre x Ai35, SST-Cre x Ai35) were recorded at normal post-CP age (P70-90), and suppression was performed by local LED illumination of V1. Paired t-test.

All error bars are SEM. *P $<0.05, * * \mathrm{P}<0.01, * * * \mathrm{P}<0.001, * * * * \mathrm{P}<0.0001$. 


\section{DISCUSSION}

In this study, we have characterized the audio-visual interaction and its developmental regulation in mouse V1. Through spike recordings, we have shown that sound modulates visual spiking activities by alternately repeating suppression and enhancement phases. This characteristic multisensory interaction depended on the effectiveness of visual orientation in each V1 neuron. Studies on other highly multisensory brain areas such as midbrain superior colliculus and association cortex revealed that the magnitude of multisensory enhancement is typically inversely related to the effectiveness of presented unimodal stimuli (Stein \& Wallace, 1996). Similarly, we found in V1 that the sound-driven spike enhancement was smaller when paired with preferred than with non-preferred orientation for each neuron. Thus, V1 also follows 'inverse effectiveness rule' of multisensory integration. Furthermore, we found that the magnitude of sound-driven spike suppression was larger with preferred orientation than with non-preferred orientation. This indicates that inverse effectiveness rule can be extended to multisensory suppression. Whether audio-visual spike interactions in V1 depend on the type of sound stimuli (Romei et al., 2012) and spatial disparity of cross-modal cues (Rohe \& Noppeney, 2016) are subject to future studies.

We also found that the sound-driven modulations of V1 activities dynamically changes around the CP. Interestingly, net sound influence on V1 spiking activities was dampened during CP due to balanced sound-driven suppression and enhancement. As a result, the orientation selectivity of V1 neurons, which was typically impaired by sounds in pre-CP and post-CP mice, was insensitive to sounds during CP. Why does V1 dampen net auditory modulations during the CP? V1 must specialize itself as visual cortex by tuning and consolidating visual selectivity of each neuron during development to efficiently process visual signals later in life. Protection of visual selectivity from sound during highly plastic CP might promote this process. On the other hand, the re-emergence of net multisensory enhancement after CP might help V1 to integrate multisensory signals after establishing its identity as visual cortex. Although the weak sounddriven modulations of V1 activity reported in our study might be subconscious to animals, it can have significant impact on the maturation of visual selectivity during $\mathrm{CP}$.

As a regulatory mechanism of sound-driven modulations, we identified a critical role of GABA-mediated inhibitions. Both field potential and spike recordings suggested the preferential maturation of sound-driven inhibitions during the CP. Furthermore, optical suppression of PV- or SST-cells revealed their involvement at different time course. Both PV- and SST-cells functionally mature during $\mathrm{CP}$, but their maturational profiles are different. For example, PVcells mature primarily by developing fast spiking and fast synaptic inhibition, while SST-cells increase their excitability without changing their spiking or output kinetics (Lazarus \& Huang, 2011). We found that early sound-driven spike suppression, which was primarily mediated by PV-cells, becomes faster and larger during CP. Therefore, the developmental profiles of early spike suppression and PV-cell are consistent with each other.

Balanced sound-driven modulations might also contribute to CP plasticity itself. A computational model proposed that improved $\mathrm{S} / \mathrm{N}$ ratio of visual modality can enhance visual plasticity in V1 (Toyoizumi et al., 2013). As a potential mechanism of the improved S/N ratio during $\mathrm{CP}$, the study showed that maturation of GABA-mediated inhibitions preferentially suppresses spontaneous activities relative to visually evoked activities. Our results extend the model and suggest that maturation of GABA-mediated inhibitions might increase the $\mathrm{S} / \mathrm{N}$ ratio of visual inputs to enhance visual plasticity both by suppressing spontaneous activities and 
balancing non-visual sensory inputs. In turn, unbalanced sound-driven modulations might contribute to dampened visual plasticity after $\mathrm{CP}$ along with the expressions of molecular brakes for visual plasticity (McGee et al., 2005; Morishita et al., 2010; Pizzorusso et al., 2002; Miyata et al., 2012). It is worth noting that the degradation time course of visual plasticity after CP (Lehmann \& Löwel, 2008) correlates well with the recovery of cAEP morphology after CP. Therefore, our findings provide a novel insight into the fundamental mechanism of circuit maturation in primary sensory cortex.

\section{EXPERIMENTAL PROCEDURES}

\section{Animals}

Founders of C57BL6/J mouse strain were purchased from Jackson laboratory. GAD65 KO, PVCre (PV-IRES-Cre), SST-Cre (Sst-IRES-Cre) and Ai35 lines were originally generated by Drs. K. Obata (Asada et al., 1996), S. Arber (Hippenmeyer et al., 2005), Z. J. Huang (Taniguchi et al., 2011) and H. Zeng (Madisen et al., 2012), respectively, and backcrossed to C57BL6/J mice. Both sexes were used. All mice were reared on $12 \mathrm{hr}$ light/dark cycle except during the darkexposure of DE mice. For dark-exposure, cages were placed in a light-tight chamber within a dark room. All procedures were approved by IACUC of Harvard University or Boston Children's Hospital.

\section{Field potential recordings}

Mice were anesthetized with Urethane $(0.8 \mathrm{~g} / \mathrm{kg}$, i.p. $)$ / Chlorprothixene $(8 \mathrm{mg} / \mathrm{kg}, \mathrm{i} . \mathrm{m}$.), and then injected with Atropine (1.5 mg/kg, s.c.) and Dexamethasone (2.5 mg/kg, s.c.). A craniotomy was made over V1, and the dura was removed before placing a chamber on V1. The chamber was filled with saline. A tungsten electrode ( 10 M $\Omega$, FST) was inserted perpendicularly into V1 $(\sim 2.5 \mathrm{~mm}$ from lambda), and the signal was band-pass filtered $(1-100 \mathrm{~Hz})$. Evoked-potentials were recorded at a depth of $\sim 250 \mu \mathrm{m}$ for all experiments. Body temperature was maintained at $38^{\circ} \mathrm{C}$, and $\mathrm{O}_{2}$ gas was continuously provided through a trachea tube. Both eyes were sutured shut during recordings to prevent potential visual inputs. White noise bursts $(80 \mathrm{~dB}, 100 \mathrm{msec}$ duration) were repeatedly presented every $2.5 \mathrm{sec}$ from a speaker placed contralaterally, and $\sim 500$ trials were averaged for each animal. Acute bath application of diazepam was performed by replacing saline in a chamber with diazepam solution (14 $\mu \mathrm{M}$ in saline). We started recordings 10 min after the diazepam application.

Data analysis was carried out using MATLAB. Evoked potentials were normalized to their prestimulus period (-200 ms to $0 \mathrm{~ms}$ ). EEGLAB toolbox (Delorme \& Makeig, 2004) was used to compute sound-driven spectral perturbations from baseline. Spectral perturbations were calculated using Morlet wavelets and normalized to their pre-stimulus periods (-500 $\mathrm{ms}$ to $0 \mathrm{~ms}$ ).

\section{Single-unit recordings}

Mice were anesthetized with Urethane $(0.8 \mathrm{~g} / \mathrm{kg}$, i.p. $) /$ Chlorprothixene $(8 \mathrm{mg} / \mathrm{kg}, \mathrm{i} . \mathrm{m}$.$) , and then$ injected with Atropine (1.5 mg/kg, s.c.) and Dexamethasone (2.5 mg/kg, s.c.). A craniotomy was made over V1, but the dura was kept intact. Spikes were recorded (Plexon) using a linear silicon probe with 16 channels (NeuroNexus Technologies). The brain was covered by $3 \%$ agarose in saline. Body temperature was maintained at $37^{\circ} \mathrm{C}$, and $\mathrm{O}_{2}$ gas continuously provided through a trachea tube. Drifting gratings ( 0.03 cycle/degree, $3 \mathrm{sec}$ duration followed by 3 sec grey screen) 
with 24 different orientations $\left(360^{\circ} / 15^{\circ}\right)$ were presented in randomized order alternately with or without a white noise burst $(80 \mathrm{~dB}, 500 \mathrm{msec}$ duration, concurrent onset with grating) from an electrostatic speaker (Tucker-Davis Technologies) placed next to contralateral ear. Ipsilateral eye was covered by a piece of black tape to limit visual inputs to contralateral eye. $\sim 10$ trials were averaged for each condition, and the average mean rate of each stimulus condition was calculated by averaging firing rates during the period of grating presentation $(3 \mathrm{sec})$. After recordings, spike waveforms were sorted by Offline Sorter (Plexon) and data analysis was performed in MATLAB.

Orientation tuning curves were drawn by fitting mean firing rates $(3 \mathrm{sec})$ to double Gaussian functions with peaks $180^{\circ}$ apart. We calculated fitting error as $E=\sum\left(R_{m}(\theta)-R_{f i t}(\theta)\right)^{2} /$ $\sum\left(R_{m}(\theta)-\bar{R}\right)^{2}$ where $R_{m}(\theta), R_{f i t}(\theta)$, and $\bar{R}$ are measured firing rate at $\theta^{\circ}$, fitted firing rate at $\theta^{\circ}$, and measured firing rate averaged across all orientations, respectively (Lee et al., 2012). Only units with [fitting error] $<0.6$ for both with and without WN sound were analyzed. OSI was defined as $O S I=\left(R_{\text {pref }}-R_{\text {ortho }}\right) /\left(R_{\text {pref }}+R_{\text {ortho }}\right)$, where $R_{\text {pref }}$ and $R_{\text {ortho }}$ are the mean firing rates of fitted tuning curves at preferred $\left(\theta_{\text {pref }}\right)$ and orthogonal $\left(\theta_{\text {ortho }}=\theta_{\text {pref }}+90^{\circ}\right)$ orientations, respectively.

For raster plots and PSTHs, $\theta_{\text {pref }}$ of each cell was defined as the orientation closest to the mean of the highest peaks of Gaussian-fitted orientation tuning curves for visual and visuo-auditory conditions. MSI was calculated as $M S I=((V A-V) / V A) * 100$ where $V$ and $V A$ are the mean firing rates (0-3 s) evoked by visual or visuo-auditory stimulus, respectively. For comparison of multisensory index among different mouse groups, we used definition of $\overline{\mathrm{MSI}}=\sum \operatorname{MSI}(\theta) / 24=$ $\sum_{\mathrm{i}=1}^{24} \operatorname{MSI}(15(\mathrm{i}-1)) / 24$ where MSIs at 24 orientations were averaged for each cell.

To draw PSTHs, firing rates were calculated using $25 \mathrm{~ms}$ bins with their centers $2 \mathrm{~ms}$ apart. Raw PSTHs were then converted to z-scores by $\frac{F R_{i}-F R_{m}}{S D_{\text {all }}}$ where $F R_{i}$ is the firing rate in the $\mathrm{i}^{\text {th }}$ bin of the PSTH, $F R_{m}$ is the mean firing rate over the entire recording period, and $S D_{\text {all }}$ is the standard deviation of firing rates for the entire recording period. Each z-score was further normalized to baseline by subtracting the mean baseline z-score value of each cell.

\section{Photo stimulation for optogenetics}

An optical fiber (200 $\mu \mathrm{m}$ diameter) was positioned $\sim 0.5 \mathrm{~mm}$ away from V1 and fixed by $3 \%$ agarose. Amber light (595 nm wavelength, 0.6-0.7 $\mathrm{mW}$ at fiber tip, Doric Lenses) was delivered through the fiber and agarose. The amber LED was turned on $0.5 \mathrm{~s}$ before visual stimulus onset and turned off $0.5 \mathrm{~s}$ after visual stimulus offset. Interleaved trials with / without amber light for each cell were compared to accurately measure cell-specific suppression.

\section{Simulation of divisive normalization model}

We used a divisive normalization model as described previously (Ohshiro et al., 2011).

Weighted linear sum of visual and auditory inputs in each neuron were defined as

$$
E=d V * I_{V}+d A * I_{A}
$$

where $d V$ is the dominance index for visual modality, $d A$ is the dominance index for auditory modality, $I_{V}$ is visual input, and $I_{A}$ is auditory input. Unisensory inputs, $\left(I_{V}, I_{A}\right)$, were given by $\sqrt{(\text { stimulus intensity) }}$ to model input nonlinearity. The activity of each neuron after divisive normalization was 


$$
R=\frac{E^{n}}{\alpha^{n}+\left(\frac{1}{N}\right) \sum_{j=1}^{N} E_{j}^{n}}
$$

where $\alpha$ is the semi-saturation constant, $N$ is the total number of neurons in the simulated network, and $n$ is the exponent for the nonlinearity between membrane potential and firing rate. For our simulations, the values of $n$ and $\alpha$ were fixed as $n=2$ and $\alpha=8$.

Our simulation was conducted using a network of 121 cells with different combinations of $d V$ and $d A(11 \times 11) . d V$ in each cell takes one of 11 values from $[0.5,0.55,0.6,0.65,0.7,0.75,0.8$, $0.85,0.9,0.95,1.0]$ while $d A$ in each cell takes one of 11 values from $[0,0.01,0.02,0.03,0.04$, $0.05,0.06,0.07,0.08,0.09,0.1]$ to simulate multisensory integration in visually dominant cortex. When V1 receives visual inputs of a specific grating orientation, some cells respond more than the others due to the heterogeneity of orientation selectivity among V1 cells. Thus, cells with large $\mathrm{dV}$ can be regarded as the cells in which the presented grating orientation is the preferred orientation, whereas cells with small $\mathrm{dV}$ can be regarded as the cells in which the presented grating orientation is non-preferred. We pooled responses of 11 cells with $\mathrm{dV}=1$ to estimate the audio-visual interaction with preferred orientation $\left(\theta_{\text {pref }}\right)$ as the stimulus and the responses of 11 cells with $\mathrm{dV}=0.5$ to estimate the audio-visual interaction with orthogonal orientation $\left(\theta_{\text {ortho }}\right)$ as the stimulus. Simulations were carried out in MATLAB.

\section{ACKNOWLEDGEMENTS}

We thank M Nakamura, E Centofante and N. De Souza for mouse colony maintenance; N Picard for advice on single-unit recording. Funded by NIMH Silvio Conte Center (1P50MH094271 to T.K.H.) and the Nakajima Foundation (R.H.).

\section{AUTHOR CONTRIBUTIONS}

R.H. designed the study, performed the experiments and simulations, interpreted the results, and wrote the manuscript. T.K.H. supervised the study.

\section{COMPETING FINANCIAL INTERESTS}

The authors declare no competing financial interests.

\section{References}

Asada, H., Kawamura, Y., Maruyama, K., Kume, H., Ding, R., Ji, F.Y., Kanbara, N., Kuzume, H., Sanbo, M., Yagi, T., and Obata, K. (1996). Mice lacking the $65 \mathrm{kDa}$ isoform of glutamic acid decarboxylase (GAD65) maintain normal levels of GAD67 and GABA in their brains but are susceptible to seizures. Biochem. Biophys. Res. Commun. 229, 891-895.

Carandini, M., and Heeger, D.J. (2011). Normalization as a canonical neural computation. Nat. Rev. Neurosci. 13, 51-62.

Charbonneau, V., Laramée, M.E., Boucher, V., Bronchti, G., and Boire, D. (2012). Cortical and subcortical projections to primary visual cortex in anophthalmic, enucleated and sighted mice. Eur. J. Neurosci. 36, 2949-2963. 
Chow, B.Y., Han, X., Dobry, A.S., Qian, X., Chuong, A.S., Li, M., Henninger, M.A., Belfort, G.M., Lin, Y., Monahan, P.E., and Boyden, E.S. (2010). High-performance genetically targetable optical neural silencing by light-driven proton pumps. Nature 463, 98-102.

Delorme, A., and Makeig, S. (2004). EEGLAB: an open source toolbox for analysis of singletrial EEG dynamics including independent component analysis. J. Neurosci. Methods 134, 9-21.

Di Cristo, G., Chattopadhyaya, B., Kuhlman, S.J., Fu, Y., Bélanger, M.C., Wu, C.Z., Rutishauser, U., Maffei, L., and Huang, Z.J. (2007). Activity-dependent PSA expression regulates inhibitory maturation and onset of critical period plasticity. Nat. Neurosci. 10, 15691577.

Espinosa, J.S., and Stryker, M.P. (2012). Development and plasticity of the primary visual cortex. Neuron 75, 230-249.

Fagiolini, M., and Hensch, T.K. (2000). Inhibitory threshold for critical-period activation in primary visual cortex. Nature 404, 183-186.

Gianfranceschi, L., Siciliano, R., Walls, J., Morales, B., Kirkwood, A., Huang, Z.J., Tonegawa, S., and Maffei, L. (2003). Visual cortex is rescued from the effects of dark rearing by overexpression of BDNF. Proc. Natl. Acad. Sci. USA 100, 12486-12491.

Gordon, J.A, and Stryker, M.P. (1996). Experience-dependent plasticity of binocular responses in the primary visual cortex of the mouse. J. Neurosci. 16, 3274-3286.

Hanover, J.L., Huang, Z.J., Tonegawa, S., and Stryker, M.P. (1999). Brain-derived neurotrophic factor overexpression induces precocious critical period in mouse visual cortex. J. Neurosci. 19, RC40.

He, H.Y., Hodos, W., and Quinlan, E.M. (2006). Visual deprivation reactivates rapid ocular dominance plasticity in adult visual cortex. J. Neurosci. 26, 2951-2955.

Hensch, T.K., Fagiolini, M., Mataga, N., Stryker, M.P., Baekkeskov, S., and Kash, S.F. (1998). Local GABA circuit control of experience-dependent plasticity in developing visual cortex. Science 282, 1504-1508.

Hensch, T. K. (2005). Critical period plasticity in local cortical circuits. Nat. Rev. Neurosci. 6, 877-888.

Hippenmeyer, S., Vrieseling, E., Sigrist, M., Portmann, T., Laengle, C., Ladle, D.R., and Arber, S. (2005). A developmental switch in the response of DRG neurons to ETS transcription factor signaling. PLoS Biol. 3, e159.

Holmes, N.P., and Spence, C. (2005). Multisensory integration: space, time and superadditivity. Curr. Biol. 2005 15, R762-R764.

Huang, Z.J., Kirkwood, A., Pizzorusso, T., Porciatti, V., Morales, B., Bear, M.F., Maffei, L., and Tonegawa, S. (1999). BDNF regulates the maturation of inhibition and the critical period of plasticity in mouse visual cortex. Cell 98, 739-755.

Huang, S., Gu, Y., Quinlan, E.M., and Kirkwood, A. (2010). A refractory period for rejuvenating GABAergic synaptic transmission and ocular dominance plasticity with dark exposure. J.

Neurosci. 30, 16636-16642. 
Iurilli, G., Ghezzi, D., Olcese, U., Lassi, G., Nazzaro, C., Tonini, R., Tucci, V., Benfenati, F., and Medini, P. (2012). Sound-driven synaptic inhibition in primary visual cortex. Neuron 73, 814-828.

Iwai, Y., Fagiolini, M., Obata, K., and Hensch, T.K. (2003). Rapid critical period induction by tonic inhibition in visual cortex. J. Neurosci. 23, 6695-6702.

Kanold, P.O., Kim, Y.A., GrandPre, T., and Shatz, C.J. (2009). Co-regulation of ocular dominance plasticity and NMDA receptor subunit expression in glutamic acid decarboxylase-65 knock-out mice. J. Physiol. 587, 2857-2867.

Kim, E.J., Juavinett, A.L, Kyubwa, E.M., Jacobs, M.W., and Callaway, E.M. (2015). Three types of cortical layer 5 neurons that differ in brain-wide connectivity and function. Neuron, 88, 12531267.

Kobayashi, Y., Ye, Z., and Hensch, T.K. (2015). Clock genes control cortical critical period timing. Neuron 86, 264-275.

Lazarus, M.S., and Huang, Z.J. (2011). Distinct maturation profiles of perisomatic and dendritic targeting GABAergic interneurons in the mouse primary visual cortex during the critical period of ocular dominance plasticity. J. Neurophysiol. 106, 775-787.

Lee, S.H., Kwan, A.C., Zhang, S., Phoumthipphavong, V., Flannery, J.G., Masmanidis, S.C., Taniguchi, H., Huang, Z.J., Zhang, F., Boyden, E.S., Deisseroth K and Dan Y. (2012).

Activation of specific interneurons improves V1 feature selectivity and visual perception. Nature 488, 379-383.

Lehmann, K., and Löwel, S. (2008). Age-dependent ocular dominance plasticity in adult mice. PLoS One 3, e3120.

Liang, M., Mouraux, A., Hu, L., and Iannetti, G.D. (2013). Primary sensory cortices contain distinguishable spatial patterns of activity for each sense. Nat. Commun. 4, 1979.

Linden, J.F., Liu, R.C., Sahani, M., Schreiner, C.E., and Merzenich, M.M. (2003).

Spectrotemporal structure of receptive fields in areas AI and AAF of mouse auditory cortex. J. Neurophysiol. 90, 2660-2675.

Lu, J., Tucciarone, J., Lin, Y., and Huang, Z.J. (2014). Input-specific maturation of synaptic dynamics of parvalbumin interneurons in primary visual cortex. Proc. Natl. Acad. Sci. USA 111, 16895-16900.

Madisen, L., Mao, T., Koch, H., Zhuo, J.M., Berenyi, A., Fujisawa, S., Hsu, Y.W., Garcia, A.J. 3rd, Gu, X., Zanella, S. Kidney J., Gu, H., Mao, Y., Hooks B.M., Boyden, E.S., Buzsáki, G., Ramirez, J.M., Jones, A.R., Svoboda, K., Han, X., Turner, E.E., Zeng, H. (2012). A toolbox of Cre-dependent optogenetic transgenic mice for light-induced activation and silencing. Nature Neurosci. 15, 793-802.

McGee, A.W., Yang, Y., Fischer, Q.S., Daw, N.W., and Strittmatter, S.M. (2005). Experiencedriven plasticity of visual cortex limited by myelin and Nogo receptor. Science 309, 2222-2226.

Miyata, S., Komatsu, Y., Yoshimura, Y., Taya, C., and Kitagawa, H. (2012). Persistent cortical plasticity by upregulation of chondroitin 6-sulfation. Nat. Neurosci. 15, 414-422. 
Morishita, H., Miwa, J.M., Heintz, N., and Hensch T.K. (2010). Lynx1, a cholinergic brake, limits plasticity in adult visual cortex. Science 330, 1238-1240.

Niell, C.M., and Stryker, M.P. (2008). Highly selective receptive fields in mouse visual cortex. J. Neurosci. 28, 7520-7536.

Ohshiro, T., Angelaki, D.E., and DeAngelis, G.C. (2011). A normalization model of multisensory integration. Nature Neurosci. 14, 775-782.

Pfeffer, C.K., Xue, M., He, M., Huang, Z.J., and Scanziani, M. (2013). Inhibition of inhibition in visual cortex: the logic of connections between molecularly distinct interneurons. Nature Neurosci. 16, 1068-1076.

Pizzorusso, T., Medini, P., Berardi, N., Chierzi, S., Fawcett, J.W., and Maffei, L. (2002). Reactivation of ocular dominance plasticity in the adult visual cortex. Science 298, 1248-1251.

Rohe, T., and Noppeney, U. (2016). Distinct Computational Principles Govern Multisensory Integration in Primary Sensory and Association Cortices. Curr. Biol. 26, 509-514.

Romei, V., Murray, M.M., Cappe, C., and Thut, G. (2009). Preperceptual and stimulus-selective enhancement of low-level human visual cortex excitability by sounds. Curr Biol. 2009 Nov 17;19(21):1799-805.Curr, Biol. 19, 1799-1805.

Romei, V., Gross, J., and Thut, G. (2012). Sounds reset rhythms of visual cortex and corresponding human visual perception. Curr. Biol. 22, 807-813.

Sugiyama, S., Di Nardo, A.A., Aizawa, S., Matsuo, I., Volovitch, M., Prochiantz, A., and Hensch T.K. (2008). Experience-dependent transfer of Otx2 homeoprotein into the visual cortex activates postnatal plasticity. Cell 134, 508-520.

Taniguchi, H., He, M., Wu, P., Kim, S., Paik, R., Sugino, K., Kvitsiani, D., Fu, Y., Lu, J., Lin, Y., Miyoshi, G., Shima, Y., Fishell, G., Nelson, S.B., and Huang, Z.J. (2011). A resource of Cre driver lines for genetic targeting of GABAergic neurons in cerebral cortex. Neuron 71, 9951013.

Toyoizumi, T., Miyamoto, H., Yazaki-Sugiyama, Y., Atapour, N., Hensch, T.K., and Miller, K.D. (2013). A theory of the transition to critical period plasticity: inhibition selectively suppresses spontaneous activity. Neuron 80, 51-63.

Vasconcelos, N., Pantoja, J., Belchior, H., Caixeta, F.V., Faber, J., Freire, M.A., Cota, V.R., Anibal de Macedo, E., Laplagne, D.A., Gomes, H.M., and Ribeiro, S. (2011). Cross-modal responses in the primary visual cortex encode complex objects and correlate with tactile discrimination. Proc. Natl. Acad. Sci. USA 108, 15408-15413.

Wallace, M.T., Ramachandran, R., and Stein B.E. (2004). A revised view of sensory cortical parcellation. Proc. Natl. Acad. Sci. USA. 101, 2167-2172.

Stein, B.E., and Wallace, M.T. (1996). Comparisons of cross-modality integration in midbrain and cortex. Prog. Brain Res. 112, 289-299.

Stodieck, S.K., Greifzu, F., Goetze, B., Schmidt, K.F., and Löwel, S. (2014). Brief dark exposure restored ocular dominance plasticity in aging mice and after a cortical stroke. Exp. Gerontol. 60, 1-11. 


\section{Supplemental figures}

A
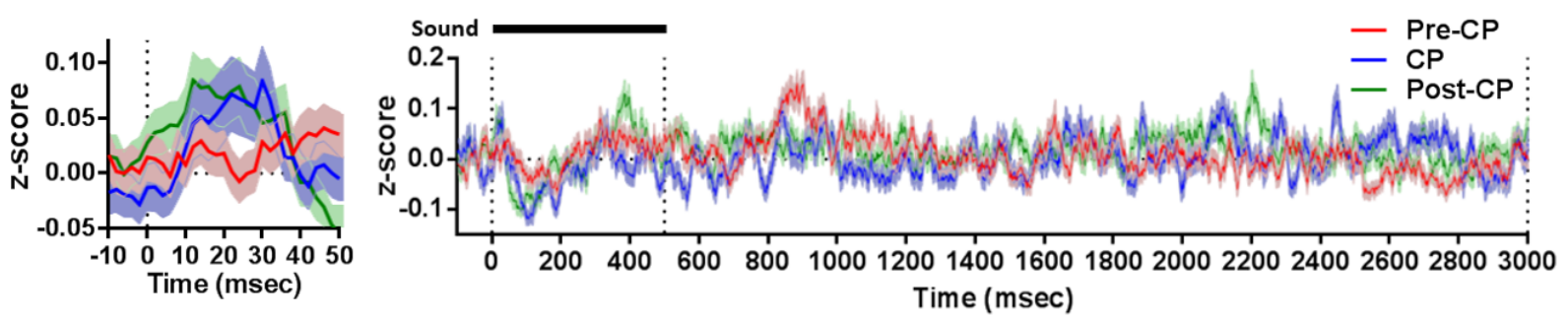

B
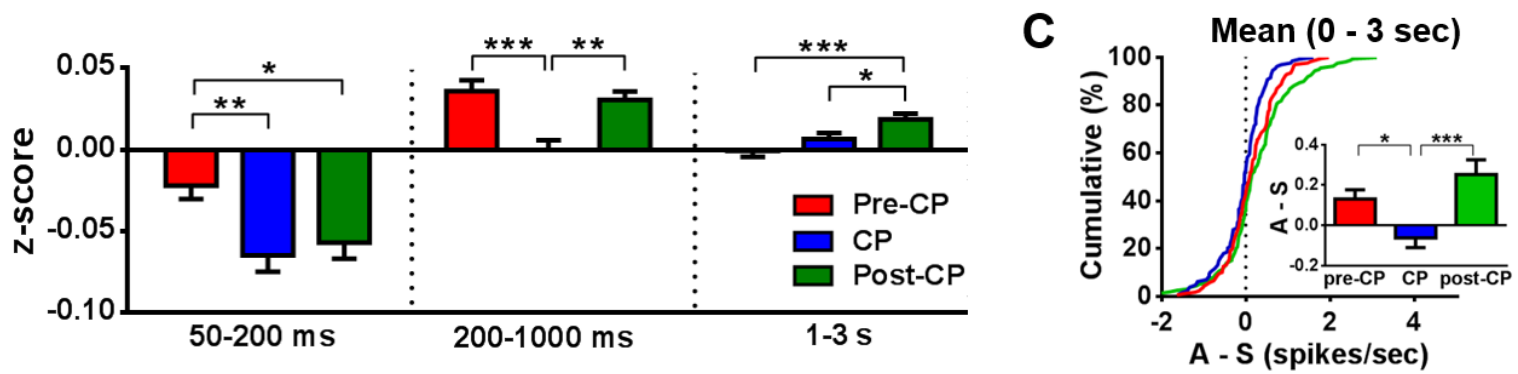

Figure S1. Sound-driven spike modulations of V1 without concurrent visual stimuli.

(A) Z-scored population averages (mean \pm SEM) of PSTHs from pre-CP (P17-19, 141 cells), CP (P26-31, 108 cells), and post-CP (P70-90, 149 cells) groups. Left panel shows the magnification of $-10-50 \mathrm{~ms}$ from the right panel to highlight the onset of sound-driven spike modulation.

(B) Auditory z-scores at different post-stimulus time intervals for different age groups.

(C) Sound-driven firing rate modulation relative to spontaneous activity. Average firing rate between 0-3 sec was calculated for auditory (A) and spontaneous (S) conditions, and they were subtracted from each other $(\mathrm{A}-\mathrm{S})$.

All error bars are SEM. $* \mathrm{P}<0.05, * * \mathrm{P}<0.01, * * * \mathrm{P}<0.001$. 


\section{A}

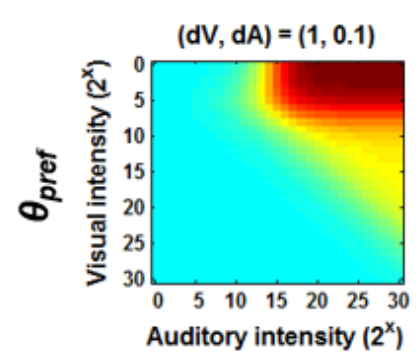

\section{Example cells}
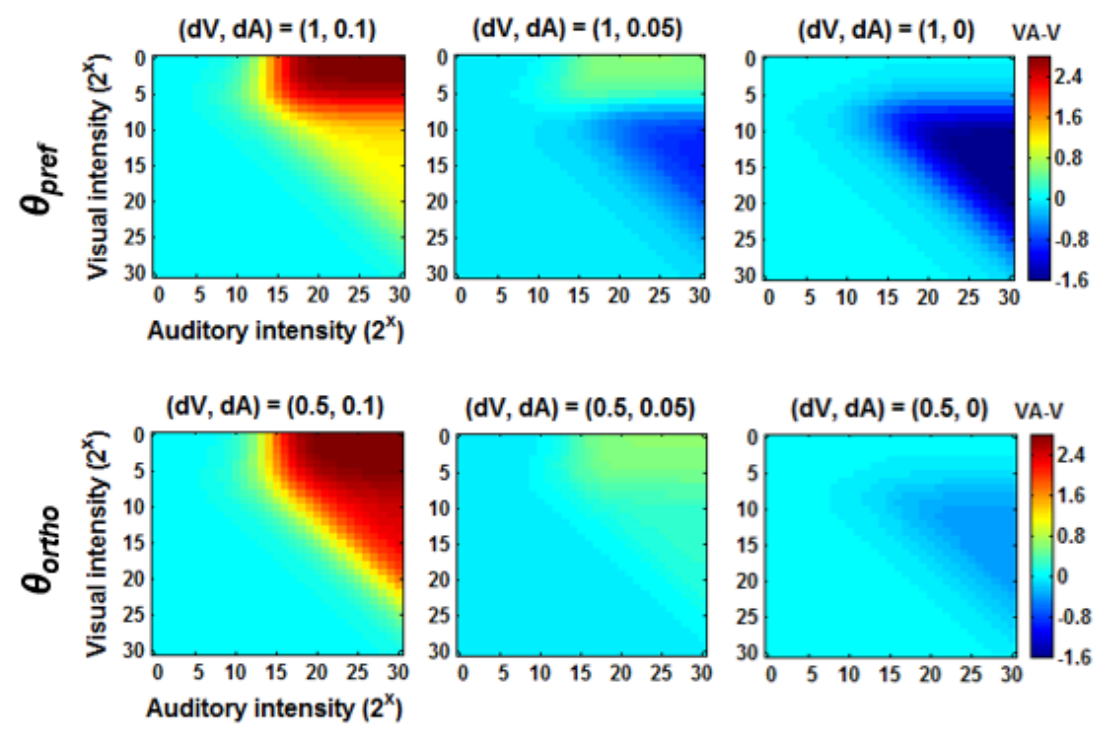

B

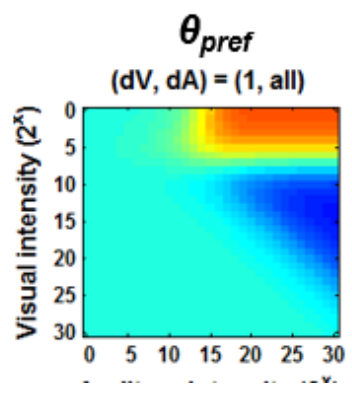

Population average
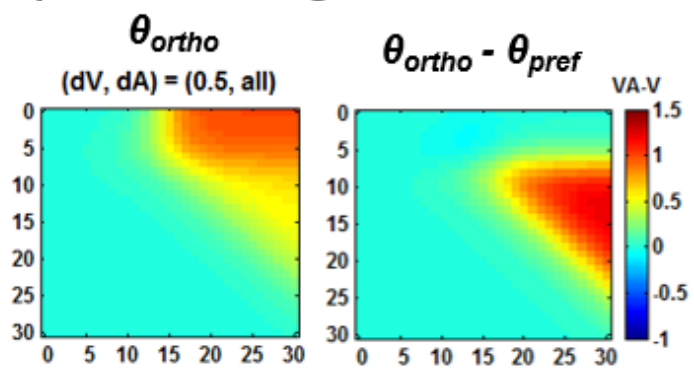

Figure S2. Divisive normalization model explains the dependence of audio-visual interaction on visual orientation.

A network of 121 cells with different combination of visual (dV) and auditory (dA) dominance index was used to simulate audiovisual interactions in visually dominant network.

(A, top row) Values of VA $-\mathrm{V}$ when paired with $\theta_{\text {pref }}(\mathrm{dV}=1.0)$ orientation are shown for three example cells with different $\mathrm{dA}$ values. VA $-\mathrm{V}$ is shown as a function of visual and auditory stimulus intensity.

(A, bottom row) Values of $\mathrm{VA}-\mathrm{V}$ when paired with $\theta_{\text {ortho }}(\mathrm{dV}=0.5)$ orientation are shown for three example cells with different $\mathrm{dA}$ values. $\mathrm{VA}-\mathrm{V}$ is shown as a function of visual and auditory stimulus intensity.

(B) Population average of 11 cells $(\mathrm{dA}=[0,0.01,0.02,0.03,0.04,0.05,0.06,0.07,0.08,0.09$, $0.1])$ with either $\mathrm{dV}=1\left(\theta_{\text {pref }}\right)$ or 0.5 . $\left(\theta_{\text {ortho }}\right)$. VA $-\mathrm{V}$ with $\theta_{\text {pref }}$ orientation and $\mathrm{VA}-\mathrm{V}$ with $\theta_{\text {ortho }}$ orientation are subtracted from each other on right panel. 